\title{
Mitochondria and the aging heart
}

\author{
Ketul R Chaudhary ${ }^{*}$, Haitham El-Sikhry ${ }^{*}$, John M Seubert \\ Faculty of Pharmacy and Pharmaceutical Sciences, University of Alberta, Edmonton, AB, T6G 2N8, Canada
}

\begin{abstract}
The average human life span has markedly increased in modern society largely attributed to advances in medical and therapeutic sciences that have successfully reduced important health risks. However, advanced age results in numerous alterations to cellular and subcellular components that can impact the overall health and function of an individual. Not surprisingly, advanced age is a major risk factor for the development of heart disease in which elderly populations observe increased morbidity and mortality. Even healthy individuals that appear to have normal heart function under resting conditions, actually have an increased susceptibility and vulnerability to stress. This is confounded by the impact that stress and disease can have over time to both the heart and vessels. Although, there is a rapidly growing body of literature investigating the effects of aging on the heart and how age-related alterations affect cardiac function, the biology of aging and underlying mechanisms remain unclear. In this review, we summarize effects of aging on the heart and discuss potential theories of cellular aging with special emphasis on mitochondrial dysfunction.
\end{abstract}

J Geriatr Cardiol 2011; 8: 159-167. doi: 10.3724/SP.J.1263.2011.00159

Keywords: aging; heart; mitochondria; heart diseases; reactive oxygen species

\section{Aging and the heart}

As the world's elderly population grows rapidly, there is an increased interest in age-related diseases, notably heart disease. By 2020, it is predicted that those older than 65 years will account for more than $20 \%$ of the total population, corresponding to the fastest growing age group worldwide. Cardiovascular diseases (CVD) are the leading causes of death in the elderly with approximately two thirds occurring after the age of 65 . This is reflected by death rates of approximately 1000 times higher in individuals who are 85-89 year old compared to those of 25-29 years of age (Table 1 and Figure 1). ${ }^{[1]}$ As such, a continued pursuit of novel therapeutic interventions to treat heart disease remains an important area of research.

Evidence from animal models and humans indicate a decreased ability of the aged heart to tolerate stress compared to young counterparts. ${ }^{[2-6]}$ Differences in how an aged heart responds to a specific disease, type of injury or individual drug therapy compared to young heart imposes

*These authors contributed equally to the manuscript.

Correspondence to: John M Seubert, $\mathrm{PhD}$, Faculty of Pharmacy and Pharmaceutical Sciences, University of Alberta, Edmonton, AB, T6G 2N8, Canada. E-mail: jseubert@pharmacy.ualberta.ca

Telephone: +1-780-492-0007

Fax: $+1-780-492-1217$

Received: July 25, 2011

Accepted: September 20, 2011

Revised: September 13, 2011

Published online: September 28, 2011

$\overline{\text { http://www.jgc301.com; jgc@mail.sciencep.com | Journal of Geriatric Cardiology }}$

an added complication. For example, an aged heart is more susceptible to adverse events and often unable to adequately overcome an adverse event. Indeed, aging causes significant reduction in the heart's ability to endure damage from ischemia and reperfusion injury. ${ }^{[4,7]}$ While aging is associated with increased oxidative stress, further increase in stress caused by injury makes the aged heart more

Table 1. Age specific mortality rate per 100,000 population.

\begin{tabular}{lcc}
\hline Age group (Years) & Major cardiovascular diseases & Diseases of heart \\
\hline $1-4$ & 0.6 & 0.4 \\
$5-9$ & 0.3 & 0.2 \\
$10-14$ & 1.0 & 0.8 \\
$15-19$ & 1.3 & 1.0 \\
$20-24$ & 1.7 & 1.3 \\
$25-29$ & 3.0 & 2.3 \\
$30-34$ & 4.6 & 3.7 \\
$35-39$ & 10.7 & 8.4 \\
$40-44$ & 21.0 & 16.7 \\
$45-49$ & 40.2 & 31.9 \\
$50-54$ & 68.0 & 54.9 \\
$55-59$ & 114.5 & 92.5 \\
$60-64$ & 191.9 & 154.8 \\
$65-69$ & 318.1 & 247.5 \\
$70-74$ & 554.3 & 410.9 \\
$75-79$ & 1027.1 & 740.9 \\
$80-84$ & 1912.7 & 1344.2 \\
$85-89$ & 3697.8 & 2591.5 \\
$>90$ & 7896.0 & 5555.3 \\
\hline
\end{tabular}




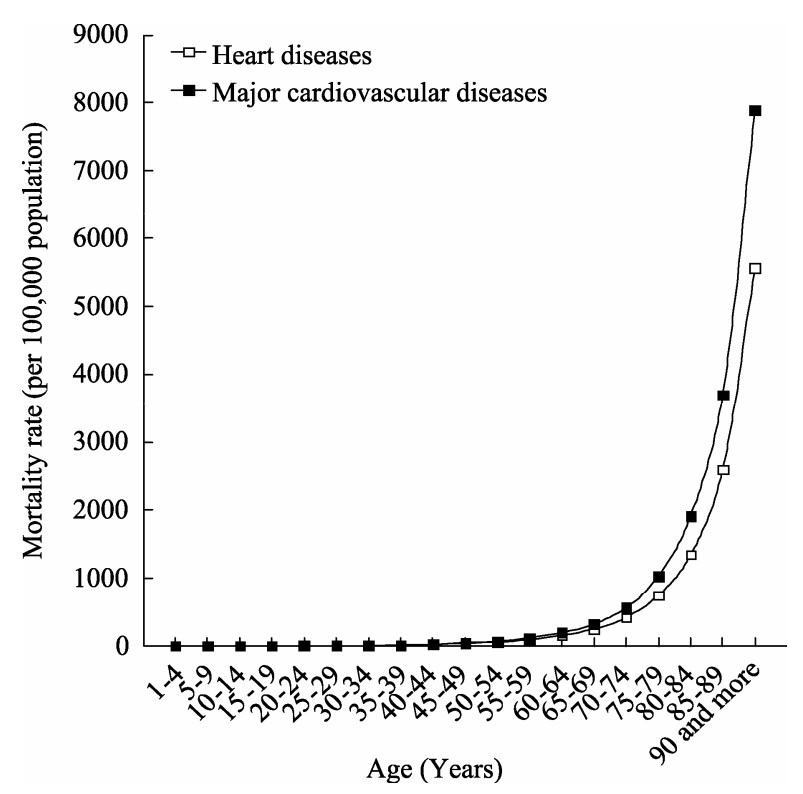

Figure 1. Age specific mortality rate.

vulnerable. ${ }^{[2,3,5,6,8,9]}$ Elderly patients often present at a clinic in an atypical manner, indicative of other diseases and complicating factors, which not only impedes diagnosis and treatment but increases susceptibility of the aged heart to more injury. ${ }^{[10]}$ Therefore, it is important to understand the biology of aging on the heart in order to determine underlying mechanisms that produce adverse effects.

\subsection{How does age affect heart function?}

\subsubsection{Diastolic function}

In Western societies, individuals over the age of 65 have the highest mortality rate due to heart failure. ${ }^{[11,12]}$ Clinical trials report that one third of these patients have an ejection fraction of more than $50 \%$, suggesting that systolic function is maintained but diastolic function is reduced. ${ }^{[13]}$ Diastolic heart failure is characterized by normal left ventricular diastolic volume, normal ejection fraction, but a delayed active relaxation and increased passive stiffness of the left ventricle. ${ }^{[14,15]}$ Abnormality of mechanical function during diastole precedes diastolic heart failure; however, this can occur with normal or abnormal systolic function and present with or without clinical signs of heart failure. ${ }^{[15,16]}$ Animal studies have demonstrated a significant reduction in contraction and relaxation velocity in aged hearts compared to younger hearts. ${ }^{[17-20]}$ In healthy individuals, there is a correlation between diastolic dysfunction and increasing age, for example, echocardiographic parameters demonstrate decreased $\mathrm{E} / \mathrm{A}$ ratio, prolonged isovolumic relaxation and early wave deceleration time occurs with age. ${ }^{[21]}$ There are numerous mechanisms proposed to cause reduced velocity and delayed relaxation such as increased myocardial wall stiffness, decreased numbers of cardiomyocytes, accumulating levels extracellular matrix and improper $\mathrm{Ca}^{+2}$ handling during action potential. ${ }^{[22,23]}$ The majority stem from molecular changes in cardiomyocyte structure and function that occur during aging.

\subsubsection{Systolic function}

In contrast to strong evidence for impaired diastolic function in aged hearts, the effect on systolic function remains mixed. Some studies have reported decreased cardiac output or decreased cardiac index while others demonstrate no significant change in either with age. ${ }^{[7,24-27]}$ The majority of studies indicate that ejection fraction, and therefore stroke volume, remains unchanged under resting conditions in an aged heart. ${ }^{[2,28]}$ Further evidence demonstrates that while cardiac index remains unaltered in aged hearts under resting conditions, the ability to increase cardiac function in response to exercise induced stress decreases with aging. ${ }^{[28]}$ Thus, suggesting systolic function is maintained at resting condition but unable to adequately respond to increased stress conditions. However, some recent studies report impaired systolic function does occur with aging. ${ }^{[20,26,29]}$ Both invasive (pressure catheter) and noninvasive (doppler echocardiography) techniques have been utilized in animal models to measure systolic dysfunction in aged mice. ${ }^{[30]}$ For example, the effects of aging on systolic function in mice demonstrated a constant decrease in left ventricular ejection fraction (LVEF) occurred with age. ${ }^{[31]}$ The decrease in LVEF was consistent with increased interstitial fibrosis, hypertrophy and activation of proapoptotic signaling. ${ }^{[31]}$ Ming et al. ${ }^{[20]}$ reported significant impairment of systolic function with age in healthy rats. In 54-week old rats, $22 \%$ decrease in $\mathrm{dp} / \mathrm{dt}_{\max }$ and $15 \%$ decrease in ejection fraction was observed compared to 9-week old rats, which clearly demonstrate age associated impairment of systolic function. ${ }^{[20]}$

\subsubsection{Heart rate}

Resting heart rates remain relatively unaffected with age; however, numerous studies have demonstrated that advancing age is associated with decreased heart rate variability (HRV). ${ }^{[24,32,33]} \mathrm{HRV}$ is defined as fluctuations in beat-to-beat interval times and is predominantly dependent on extrinsic regulators. HRV reflects the heart's ability to respond and adapt to changing extracardiac factors such as stress. ${ }^{[34]}$ Higher HRV indicates a quicker ability to change the heart rate in response to stress conditions, thus, a lower HRV in aged hearts results in a reduced ability to respond to appropriately. ${ }^{[32,33]}$ Reports suggest a decreased responsiveness to $\beta$-adrenergic receptor in aged hearts might be responsible 
for decreased HRV in aged people during exercise or stress. ${ }^{[35]}$

\subsection{What leads to impaired function in aged hearts?}

\subsubsection{Changes in ventricle structure}

Alterations to heart structure attributed to age-dependent effects is thought to significantly impact overall heart function. ${ }^{[36-38]}$ Evidence suggests that there is an increase in left ventricular mass with aging, in which heart weight can increase $1 \mathrm{~g}$ to $1.5 \mathrm{~g}$ per year. ${ }^{[39,40]}$ Although, studies that exclude individuals with heart diseases fail to report significant changes in left ventricular mass. ${ }^{[36,37]}$ Conversely, an increase in relative wall thickness has been reported in aged hearts from healthy individuals. ${ }^{[41]}$ The increased wall thickness is accompanied by decreased cardiomyocyte count and increased left ventricular collagen content, which are features of fibrosis. ${ }^{[42]}$ Animal studies have demonstrated left ventricular collagen content increases linearly with age but can increase two-fold during the later years of life. ${ }^{[42]}$ Overall, the cumulative impact of decreased cardiomyocyte count, increased collagen deposition and increased stiffness leads to significant ventricular dysfunction which is a major characteristic of the aging heart. ${ }^{[37,43]}$

\subsubsection{Changes at the cellular level}

Age-dependent changes to left ventricular structure and function are associated with increased fibrosis leading to ventricular stiffening and diastolic dysfunction. With increased collagen content and increased stiffness, cardiomyocyte hypertrophy contributes significantly towards diastolic dysfunction in aged heart. At the cellular level, heart structure is comprised of numerous cell types that make up three main components: cardiac muscle tissue, the conduction system and the extracellular connective tissue, which consists mainly of collagen. Cardiac muscle cells, or cardiomyocytes, are the contractile cells, which form a major part of the ventricular and atrial walls. Previously, it was thought that cardiomyocytes were terminally differentiated and their lifespan was the same as the heart. However, identification of resident cardiac stem cells and data demonstrating that cardiomyocytes can regenerate suggest myocyte turnover exists. ${ }^{[44,45]}$ Still the extent of cardiomyocyte renewal remains controversial as reports range from $0 \%-20 \%$ renewal every year. ${ }^{[44]}$ The fact cardiomyocytes are formed postnatally and significantly decrease in number with age, suggests the rate of cardiomyocyte regeneration decreases and/or any regeneration cannot compensate for cell loss over time. ${ }^{[37,44]}$ The loss of cardiomyocytes in aged hearts is then compensated by increase in cell size or by cellular hypertrophy. ${ }^{[37]}$
Importantly, aging also affects the structure and function of cardiac interstitial cells, such as endothelial and fibroblasts that play an important role in the pathogensis of CVD.

Extensive evidence demonstrates aging directly influences the expression and function of various ion channels, receptors, enzymes and signaling molecules that significantly impact heart function. ${ }^{[35,46-48]}$ For example, advanced age is associated with alterations in expression of sarcoplasmic reticulum calcium ATPase (SERCA) and phospholambam, resulting in improper regulation of intracellular $\mathrm{Ca}^{2+}$ leading to prolonged activation of contractile proteins in cardiac muscles. ${ }^{[46]}$ This effect can be evident in the form of prolonged isovolumetric relaxation in aged human hearts. ${ }^{[46]}$ Moreover, increased intracellular $\mathrm{Ca}^{2+}$ for a prolonged period causes increased $\mathrm{Ca}^{2+}$ uptake by mitochondria, which further leads to mitochondrial dysfunction and cardiomyocyte death. Similarly, expression and/or function of ATP sensitive $\mathrm{K}^{+}\left(\mathrm{K}_{\mathrm{ATP}}\right)$ channels decreases with advanced age affecting the electrical activities of cardiomyocytes. ${ }^{[47-49]}$ Decreased $\mathrm{K}_{\text {ATP }}$ channel density represents a possible mechanism for decreased tolerance of aged heart against ischemic injury. ${ }^{[47,48]}$ Overall, numerous age-related effects to cellular and subcellular structure and function of key channels, proteins and molecules play an essential role in the pathogenesis of CVD in aged individuals.

\section{Aging and mitochondria}

In the heart, mitochondria provide the primary source of energy that fuels the contractile apparatus and act as key regulators of cell survival and death. These dynamic organelles undergo continuous fusion and fission processes (dynamics), which are closely related to cellular energy demands and stress levels. ${ }^{[50]}$ Impaired mitochondrial function over time has been suggested as an underlying cause of cardiomyocyte loss during aging. ${ }^{[51]}$ Reports suggest that bio-energetically efficient mitochondria from young individuals become less numerous, swollen, and chronically depolarized. ${ }^{[51]}$ Importantly, mitochondrial integrity is vital for cellular homeostasis and cardiac performance.

\subsection{Mitochondrial theory of cellular aging}

The connection between mitochondria and aging started in 1972 when Harman et al. ${ }^{[52]}$ proposed the role of mitochondria as the main source of reactive oxygen species (ROS) and free radicals, which are mediators in cellular age-dependant damage. In 1980, Miquel and his colleagues refined this role emphasizing that mitochondria are the main source of ROS and the main target of their injury, 
suggesting they were the key "organelles" initiating cellular processes leading to aging. ${ }^{[53]}$

Mitochondrial theory of cellular aging relies on the fact that mitochondrial DNA (mtDNA) has a high rate of mutation and limited capacity for repair. ${ }^{[54]}$ Over time, accumulation of mtDNA mutations compromises the integrity of the mitochondrial genome which is essential for mitochondrial function. ${ }^{[54]}$ Thus, mitochondria gradually lose their ability to produce energy while continue to emit higher amount of ROS. ${ }^{[55]}$ As the tissue ages, populations of dysfunctional mitochondria in the cell increases, especially in terminally differentiated non-proliferative organs such as brain and heart, causing energy shortage and a growing oxidative burden. This leads to cellular damage or death then tissue degeneration and dysfunction, which are seen in age-related diseases that eventually kill the individual. In short, this theory considers mtDNA and its mutation rates as the "aging clock" that initiates aging events influencing the overall organism longevity.

Rates of mtDNA mutation have been reported to be 15-fold higher than that of nuclear DNA (nDNA), which lead to aberrant expression of electron transport chain (ETC) proteins impairing the oxidative phosphorylation process (OxPhos). ${ }^{[56,57]}$ These age-dependent changes in mitochondria result in larger and less numerous organelles with lower mitochondrial membrane potential and ultra structural abnormalities. $^{[58]}$ Importantly, ETC dysfunction has been demonstrated to decrease ATP production and increase ROS emission as well impair nucleotide synthesis, that, in turn, affects nDNA genes. ${ }^{[59]}$ Our current level of knowledge suggests that the decline of mitochondrial integrity and function is implicated in age related and life limiting diseases such as heart failure, diabetes, cancer and neurodegeneration. ${ }^{[60,61]}$

\subsubsection{Aging and mtDNA}

Bacteria-like features of mitochondria support the hypothesis of their evolutionary origin from bacteria and notion of conserved aging pathways between species. ${ }^{\text {[62-65] }}$ Mitochondria are unique organelles that contain their own DNA, transcriptional and translational synthesis machinery. However, mitochondria cannot be synthesized de novo, instead they replicate in the cytosolic compartment through a process of division. ${ }^{[66]}$ Human mtDNA contain 37 genes encoding 13 polypeptides that compose subunits within the OxPhos complexes. The remaining mitochondrial proteins are encoded in nDNA genes, including 80 OxPhos genes, mtDNA polymerase $\gamma$, mtDNA transcription factors and metabolic pathways related genes. ${ }^{[67-70]}$ Importantly the proteins that are encoded by mtDNA are vital for normal mitochondrial function. ${ }^{[59]}$

mtDNA does not have the protein protection that histones provide to $\mathrm{nDNA}$ and has less effective repair mechanisms. ${ }^{[59,71]}$ Mutations in mtDNA affecting the expression and integrity of OxPhos complexes can cause mitochondrial dysfunction and increased ROS production. ${ }^{[69]}$ Conversely, located in the mitochondrial matrix, mtDNA is exposed to these destructive ROS byproducts making it highly prone to oxidative damage. ${ }^{[59]}$ The amount of 8-hydroxydeoxyguanosine, marker of DNA oxidative damage, found in mtDNA was found to be 16 times higher than the corresponding ratio in nDNA in rat liver cells. ${ }^{[71]}$ Resultant mutations can be transferred during mitochondrial division to daughter mitochondria producing sub-populations of mutant mitochondria within a cell. ${ }^{[72]}$ Cellular mtDNA mutations tend to persist and accumulate resulting in impaired mitochondrial metabolism, ROS production and defective cellular functions. ${ }^{[73,74]}$ Together, this results in a wide range of pathological abnormalities, which depends upon the severity of mtDNA alterations, amount of dysfunctional mitochondria and the specific tissue impacted. ${ }^{[75,76]}$ Indeed, progressive accumulation of mtDNA mutations has been associated with age-related deterioration of function in tissues and organs such as heart, nervous system and kidney. ${ }^{[69]}$

It is uncertain whether mtDNA deterioration is an independent "cause" in the aging process or mtDNA mutations is merely an "effect" of the increased oxidative damage and overall functional decline in the aging cell. Development of a mouse model with defective mtDNA polymerase $\gamma$ (POLG) has provided evidence for a role of mutated mtDNA in aging. ${ }^{[77]}$ The designed POLG was defectively proofreading mtDNA during replication leading to higher introduction rate of random mutations. As a result, the level of mtDNA point mutations increased three to five folds along with broken linear mtDNA and deletions. These "mtDNA mutator mice" were healthy and normal up to their early adolescence, but then exhibited a clear premature onset of aging phenotypes such as osteoporosis, weight loss, heart enlargement, anemia, curvature of the spine, hair loss, and reduced fertility, associated with significant reduction of life span.

\subsubsection{Aging and mitochondrial ROS}

Evidence suggests that ROS and free radicals act as major mediators responsible for the cellular damage seen in aged cells. ${ }^{[78]}$ High concentrations of ROS cause irreversible oxidative damage to proteins, nucleic acids and membrane lipids, causing general decline of cell function. ${ }^{[59,61]}$ Cellular 
antioxidant enzymes and ROS scavengers provide protection by maintaining their levels within beneficial ranges. ${ }^{[61,65]}$ Accordingly, oxidative stress can result from either elevated ROS generation or reduced antioxidant capacity. Data from genetically altered mice with disrupted p66shc gene, an adaptor protein responsible for mitochondrial ROS generation independent from OxPhos process, showed an increased resistance to oxidative stress associated with $30 \%$ extension in lifespan. ${ }^{[79]}$ Further evidence for a marked lifespan extension is observed in transgenic mice overexpressing either the mitochondrial antioxidant enzyme manganese superoxide dismutase (MnSOD) or catalase. ${ }^{[80,81]}$ Moreover, mice deficient in MnSOD demonstrate early perinatal death associated with severe mitochondrial damage, dilated cardiomyopathy and neurodegeneration. ${ }^{[82,83]}$ While these studies demonstrate a link between ROS levels and longevity, they also indicate the importance of mitochondria as a primary target for oxidative damage. Consequently, modification of lifespan in transgenic models with modulated oxidative stress levels can be explained by regulation of mtDNA mutation rate.

An important issue is the relationship between ROS levels and mtDNA mutations. It has been proposed that the progressive mutations in mtDNA and the resultant decline in mitochondrial activity observed in aged tissues, ${ }^{[84,85]}$ is responsible for the increased generation of ROS, which, in turn, will attack mitochondria causing further mtDNA damage. This "vicious cycle" concept ${ }^{[86,87]}$ postulated that accumulation of mtDNA mutations is exponential and associated with massive increase in ROS production. ${ }^{[61]}$ However, the vicious cycle hypothesis has been challenged by evidence from the mtDNA mutator mice. These mice exhibit a linear accumulation of mtDNA mutations over their lifespan. However, although they have severe respiratory chain dysfunction, there is no significant increase in ROS levels and normal expression of antioxidant enzymes, such as MnSOD and glutathione peroxidase 1, compared to wild type controls. ${ }^{[88]}$ These results strongly argue that mtDNA mutation and the respiratory chain dysfunction are the primary inducer of premature aging in these mice independent from the downstream role of ROS. ${ }^{[88]}$

\subsection{Mitochondrial quality control}

Mitochondria are strategic organelles essential for cell function and homeostasis, which provide energy and act as key regulators of cell death. Indeed, mitochondrial dysfunction is repeatedly reported in aged tissues. ${ }^{[57,85,89,90]}$ Impairment in mitochondrial integrity, dynamics or metabolic activity results in a range of deleterious effects to the cell such as reduced ATP production, elevated cytosolic calcium, increased ROS release and release of proapoptotic factors triggering cell death. ${ }^{[59,91-94]}$ Dysfunctional mitochondria can trigger removal of damaged cells via apoptosis; however, opposite to proliferating tissues, this can be severely detrimental in postmitotic non-proliferative tissues, such as brain and heart. For example, in cardiomyocytes, which are long-living cells with no dividing ability and highly dependent upon mitochondria for energy production, mitochondria form approximately $30 \%$ of the total cell volume and approximately $90 \%$ of the required energy. ${ }^{[95]}$ Therefore, an efficient quality control system is essential to remove dysfunctional mitochondria and maintain their overall integrity. Impairment to this system will severely impact cardiac homeostasis.

Mitochondrial quality control is composed of two processes: one, removal of defective mitochondria by autophagy, and, two, biogenesis of new mitochondria. In autophagy, the cell deliberately digests and recycles damaged components such as proteins and organelles to increase survivability. ${ }^{[96,97]}$ Experimental evidence demonstrates that loss-of-function mutations in genes encoding autophagic proteins, such as Atg5, resulting in suppression of basal autophagy leading to a significant shortening of lifespan. ${ }^{[98-100]}$ Aging is associated with a decline in autophagy, which may explain the accumulation of aberrant organelles and macromolecules in senescent cells. ${ }^{[61,97,101,102]}$ For example, mitochondria with inhibited respiratory chain and depolarized membrane are unable to fuse with other healthy mitochondria then they are targeted for removal by autophagy. ${ }^{[103]}$ Twig et al. ${ }^{[104]}$ observed that a single mitochondrion can undergo mitochondrial fission into two daughter mitochondria; a functionally active polarized mitochondrion and a non-functional depolarized mitochondrion. The non-functional mitochondrion is subsequently removed by autophagy suggesting a segregation process among individual mitochondrion, which retains healthy mitochondria with more efficient energy production and better resistance to cellular stress. Caloric restriction is shown to increase both median and mean lifespan in several models from yeast to mammals. ${ }^{[105]}$ Interestingly, caloric restriction has been demonstrated to induce autophagic pathways ${ }^{[106]}$ and mitochondrial biogenesis by activating the transcriptional modulator peroxisome proliferator-activated receptor coaltivator-1 $\alpha$ (PGC-1 $\alpha) .{ }^{[61,67]}$ Both are key components of mitochondrial quality control that lead to removal of dysfunctional mitochondria and enhanced longevity. ${ }^{[106]}$

\section{Conclusion}

Aging is a natural process that occurs in all organisms; however, the underlying mechanisms and biology of this 
complex phenotype remains incompletely understood. CVD is a major cause of disability and mortality in elderly individuals in which the rates of incidence rapidly rise after the age of 65. Aging results in an intricate series of alterations to heart structure and function adapting to meet the needs of an older body. Even healthy individuals that appear to have normal heart function under resting conditions, actually have an increased susceptibility and vulnerability to stress. This is confounded by the impact that stress and disease can have over time to both the heart and vessels. There is a rapidly growing body of literature investigating the effects of aging on the heart and how age-related alterations to gene expression, protein modification and tissue structure effect cardiac function. Recent advances into understanding the role of mitochondria in maintaining cardiac energetics and survival are providing new ideas for developing novel therapies to limit and prevent susceptibility to injury in the aged heart.

\section{Acknowledgment}

Chaudhary KR is recipient of a doctoral research award from the Heart and Stroke Foundation of Canada and a Studentship Award from Alberta Innovates Health Solutions. El-Sikhry $\mathrm{H}$ is the recipient of a studentship award from Alberta Innovates Health Solutions. Seubert JM is the recipient of a new investigator award from the Heart and Stroke Foundation of Canada, a health scholar award from the Alberta Heritage Foundation for Medical Research (AHFMR). This work was supported by an operating grant from Heart and Stroke Foundation of Canada (JMS).

\section{References}

1 Statistics-Canada. Mortality, Summary List of Causes. 2007. www.statcan.gc.ca/pub/84f0209x/84f0209x2007000-eng.htm (accessed on September 15, 2011).

2 Starnes JW, Bowles DK, Seiler KS. Myocardial injury after hypoxia in immature, adult and aged rats. Aging (Milano) 1997; 9: 268-276.

3 Lesnefsky EJ, Gallo DS, Ye J, et al. Aging increases ischemia- reperfusion injury in the isolated, buffer-perfused heart. J Lab Clin Med 1994; 124: 843-851.

4 Liu M, Zhang $\mathrm{P}$, Chen M, et al. Aging might increase myocardial ischemia/reperfusion-induced apoptosis in humans and rats. Age (Dordr) 2011; In press.

5 Mariani J, Ou R, Bailey M, et al. Tolerance to ischemia and hypoxia is reduced in aged human myocardium. $J$ Thorac Cardiovasc Surg 2000; 120: 660-667.

6 Rosenfeldt FL, Pepe S, Ou R, et al. Coenzyme Q10 improves the tolerance of the senescent myocardium to aerobic and ischemic stress: studies in rats and in human atrial tissue. Biofactors 1999; 9: 291-299.

7 Abete P, Napoli C, Santoro G, et al. Age-related decrease in cardiac tolerance to oxidative stress. J Mol Cell Cardiol 1999; 31: 227-236.

8 Jansen-Durr P, Osiewacz HD. Healthy ageing: a question of stress, damage and repair. Meeting on mechanisms of biological ageing. EMBO Rep 2002; 3: 1127-1132.

9 Misare BD, Krukenkamp IB, Levitsky S. Age-dependent sensitivity to unprotected cardiac ischemia: the senescent myocardium. J Thorac Cardiovasc Surg 1992; 103: 60-64.

10 Jugdutt BI. Aging and heart failure: changing demographics and implications for therapy in the elderly. Heart Fail Rev 2010; 15: 401-405.

11 Ahmed A. Chronic heart failure in older adults. Med Clin North Am 2011; 95: 439-461.

12 Roger VL, Go AS, Lloyd-Jones DM, et al. Heart disease and stroke statistics - 2011 update: a report from the American Heart Association. Circulation 2011; 123: e18-e209.

13 Bhatia RS, Tu JV, Lee DS, et al. Outcome of heart failure with preserved ejection fraction in a population-based study. N Engl J Med 2006; 355: 260-269.

14 Wood P, Piran S, Liu PP. Diastolic heart failure: progress, treatment challenges, and prevention. Can J Cardiol 2011; 27: 302-310.

15 Zile MR, Brutsaert DL. New concepts in diastolic dysfunction and diastolic heart failure: Part I: diagnosis, prognosis, and measurements of diastolic function. Circulation 2002; 105: 1387-1393.

16 Shibata S, Hastings JL, Prasad A, et al. Congestive heart failure with preserved ejection fraction is associated with severely impaired dynamic Starling mechanism. J Appl Physiol 2011; 110: 964-971.

17 Yang X, Sreejayan N, Ren J. Views from within and beyond: narratives of cardiac contractile dysfunction under senescence. Endocrine 2005; 26: 127-137.

18 Yang X, Doser TA, Fang CX, et al. Metallothionein prolongs survival and antagonizes senescence-associated cardiomyocyte diastolic dysfunction: role of oxidative stress. FASEB J 2006; 20: 1024-1026.

19 Shinmura K, Tamaki K, Sano M, et al. Impact of long-term caloric restriction on cardiac senescence: caloric restriction ameliorates cardiac diastolic dysfunction associated with aging. J Mol Cell Cardiol 2011; 50: 117-127.

20 Ming Z, Legare DJ, Lautt WW. Absence of meal-induced insulin sensitization (AMIS) in aging rats is associated with cardiac dysfunction that is protected by antioxidants. $J$ Appl Physiol 2011; In press. 
21 Wierzbowska-Drabik K, Krzeminska-Pakula M, Chrzanowski L, et al. Age-dependency of classic and new parameters of diastolic function. Echocardiography 2008; 25: 149-155.

22 Sanders D, Dudley M, Groban L. Diastolic dysfunction, cardiovascular aging, and the anesthesiologist. Anesthesiol Clin 2009; 27: 497-517.

23 Fares E, Howlett SE. Effect of age on cardiac excitation-contraction coupling. Clin Exp Pharmacol Physiol 2010; 37: 1-7.

24 Onose Y, Oki T, Mishiro Y, et al. Influence of aging on systolic left ventricular wall motion velocities along the long and short axes in clinically normal patients determined by pulsed tissue doppler imaging. J Am Soc Echocardiogr 1999; 12: 921-926.

25 Bujak M, Kweon HJ, Chatila K, et al. Aging-related defects are associated with adverse cardiac remodeling in a mouse model of reperfused myocardial infarction. $\mathrm{J} \mathrm{Am} \mathrm{Coll} \mathrm{Cardiol}$ 2008; 51: 1384-1392.

26 Slotwiner DJ, Devereux RB, Schwartz JE, et al. Relation of age to left ventricular function in clinically normal adults. $\mathrm{Am}$ J Cardiol 1998; 82: 621-626.

27 Shannon RP, Maher KA, Santinga JT, et al. Comparison of differences in the hemodynamic response to passive postural stress in healthy subjects greater than 70 years and less than 30 years of age. Am J Cardiol 1991; 67: 1110-1116.

28 Fleg JL, O'Connor F, Gerstenblith G, et al. Impact of age on the cardiovascular response to dynamic upright exercise in healthy men and women. $J$ Appl Physiol 1995; 78: 890-900.

29 Ahmet I, Tae HJ, de Cabo R, et al. Effects of calorie restriction on cardioprotection and cardiovascular health. $J$ Mol Cell Cardiol 2011; 51: 263-271.

30 Reddy AK, Amador-Noguez D, Darlington GJ, et al. Cardiac function in young and old Little mice. J Gerontol A Biol Sci Med Sci 2007; 62: 1319-1325.

31 Boyle AJ, Shih H, Hwang J, et al. Cardiomyopathy of aging in the mammalian heart is characterized by myocardial hypertrophy, fibrosis and a predisposition towards cardiomyocyte apoptosis and autophagy. Exp Gerontol 2011; 46: 549-559.

32 De Meersman RE. Heart rate variability and aerobic fitness. Am Heart J 1993; 125: 726-731.

33 Carter JB, Banister EW, Blaber AP. The effect of age and gender on heart rate variability after endurance training. Med Sci Sports Exerc 2003; 35: 1333-1340.

34 Romanowicz M, Schmidt JE, Bostwick JM, et al. Changes in heart rate variability associated with acute alcohol consumption: current knowledge and implications for practice and research. Alcohol Clin Exp Res 2011; 35: 1092-1105.
35 Xiao RP, Spurgeon HA, O'Connor F, et al. Age-associated changes in beta-adrenergic modulation on rat cardiac excitation-contraction coupling. J Clin Invest 1994; 94: 2051-2059.

36 Cheng S, Fernandes VR, Bluemke DA, et al. Age-related left ventricular remodeling and associated risk for cardiovascular outcomes: the multi-ethnic study of atherosclerosis. Circ Cardiovasc Imaging 2009; 2: 191-198.

37 Olivetti G, Melissari M, Capasso JM, et al. Cardiomyopathy of the aging human heart. Myocyte loss and reactive cellular hypertrophy. Circ Res 1991; 68: 1560-1568.

38 Olivetti G, Cigola E, Maestri R, et al. Aging, cardiac hypertrophy and ischemic cardiomyopathy do not affect the proportion of mononucleated and multinucleated myocytes in the human heart. J Mol Cell Cardiol 1996; 28: 1463-1477.

39 Melissari M, Balbi T, Gennari M, et al. The aging of the heart: weight and structural changes in the left ventricle with age. $G$ Ital Cardiol 1991; 21: 119-130.

40 Linzbach AJ, Akuamoa-Boateng E. Changes in the aging human heart. I. Heart weight in the aged. Klin Wochenschr 1973; 51: 156-163.

41 Hees PS, Fleg JL, Lakatta EG, et al. Left ventricular remodeling with age in normal men versus women: novel insights using three-dimensional magnetic resonance imaging. Am J Cardiol 2002; 90: 1231-1236.

42 Sangaralingham SJ, Huntley BK, Martin FL, et al. The aging heart, myocardial fibrosis, and its relationship to circulating C-type natriuretic peptide. Hypertension 2011; 57: 201-207.

43 Burlew BS, Weber KT. Connective tissue and the heart. Functional significance and regulatory mechanisms. Cardiol Clin 2000; 18: 435-442.

44 Kajstura J, Urbanek K, Perl S, et al. Cardiomyogenesis in the adult human heart. Circ Res 107: 305-315.

45 Bergmann O, Bhardwaj RD, Bernard S, et al. Evidence for cardiomyocyte renewal in humans. Science 2009; 324: 98-102.

46 Fares E, Howlett SE. Effect of age on cardiac excitation-contraction coupling. Clin Exp Pharmacol Physiol 2010; 37: $1-7$.

47 Jovanovic S, Jovanovic A. Sarcolemmal K(ATP) channels in ageing. Ageing Res Rev 2004; 3: 199-214.

48 Ranki HJ, Crawford RM, Budas GR, et al. Ageing is associated with a decrease in the number of sarcolemmal ATP-sensitive $\mathrm{K}+$ channels in a gender-dependent manner. Mech Ageing Dev 2002; 123: 695- 705.

49 Raveaud S, Verdetti J, Faury G. Nicorandil protects ATP-sensitive potassium channels against oxidation-induced dysfunction in cardiomyocytes of aging rats. Biogerontology 2009; 10: 537-547. 
50 Chan DC. Mitochondria: dynamic organelles in disease, aging, and development. Cell 2006; 125: 1241-1252.

51 Hafner AV, Dai J, Gomes AP, et al. Regulation of the mPTP by SIRT3-mediated deacetylation of CypD at lysine 166 suppresses age-related cardiac hypertrophy. Aging (Albany NY) 2010; 2: 914-923.

52 Harman D. The biologic clock: the mitochondria? $\mathrm{J} \mathrm{Am}$ Geriatr Soc 1972; 20: 145-147.

53 Miquel J, Economos AC, Fleming J, et al. Mitochondrial role in cell aging. Exp Gerontol 1980; 15: 575-591.

54 Linnane AW, Marzuki S, Ozawa T, et al. Mitochondrial DNA mutations as an important contributor to ageing and degenerative diseases. Lancet 1989; 1: 642-645.

55 Gottlieb RA, Gustafsson AB. Mitochondrial turnover in the heart. Biochim Biophys Acta 2011; 1813: 1295-1301.

56 Short KR, Bigelow ML, Kahl J, et al. Decline in skeletal muscle mitochondrial function with aging in humans. Proc Natl Acad Sci USA 2005; 102: 5618-5623.

57 Boffoli D, Scacco SC, Vergari R, et al. Decline with age of the respiratory chain activity in human skeletal muscle. Biochim Biophys Acta 1994; 1226: 73-82.

58 Frenzel H, Feimann J. Age-dependent structural changes in the myocardium of rats: A quantitative light- and electron-microscopic study on the right and left chamber wall. Mech Ageing Dev 1984; 27: 29-41.

59 Desler C, Marcker ML, Singh KK, et al. The importance of mitochondrial DNA in aging and cancer. J Aging Res 2011; 407536.

60 Chen L, Gong Q, Stice JP, et al. Mitochondrial OPA1, apoptosis, and heart failure. Cardiovasc Res 2009; 84: 91-99.

61 Raffaello A, Rizzuto R. Mitochondrial longevity pathways. Biochim Biophys Acta 2011; 1813: 260-268.

62 Sagan L. On the origin of mitosing cells. J Theor Biol 1967; 14: 255-274.

63 Margulis L. Symbiotic theory of the origin of eukaryotic organelles; criteria for proof. Symp Soc Exp Biol 1975; (29): 21-38.

64 Margulis L. Recombination of non-chromosomal genes in Chlamydomonas: assortment of mitochondria and chloroplasts? J Theor Biol 1970; 26: 337-342.

65 Balaban RS, Nemoto S, Finkel T. Mitochondria, oxidants, and aging. Cell 2005; 120: 483-495.

66 Hom J, Sheu SS Morphological dynamics of mitochondriaA special emphasis on cardiac muscle cells. $\mathrm{J} \mathrm{Mol} \mathrm{Cell}$ Cardiol 2009; 46: 811-820.

67 Vendelbo MH, Nair KS. Mitochondrial longevity pathways. Biochim Biophys Acta 2011; 1813: 634-644.

68 Wallace DC. Mitochondrial genetics: a paradigm for aging and degenerative diseases? Science 1992; 256: 628-632.
69 Wallace DC. Mitochondrial DNA mutations in disease and aging. Environ Mol Mutagen 2010; 51: 440-450.

70 Wallace DC. A mitochondrial paradigm of metabolic and degenerative diseases, aging, and cancer: a dawn for evolutionary medicine. Annu Rev Genet 2005; 39: 359-407.

71 Richter C, Park JW, Ames BN. Normal oxidative damage to mitochondrial and nuclear DNA is extensive. Proc Natl Acad Sci USA 1988; 85: 6465-6467.

72 Yakes FM, Van Houten B. Mitochondrial DNA damage is more extensive and persists longer than nuclear DNA damage in human cells following oxidative stress. Proc Natl Acad Sci USA 1997; 94: 514-519.

73 Larsson NG, Clayton DA. Molecular genetic aspects of human mitochondrial disorders. Annu Rev Genet 1995; 29: 151-178.

74 Fayet G, Jansson M, Sternberg D, et al. Ageing muscle: clonal expansions of mitochondrial DNA point mutations and deletions cause focal impairment of mitochondrial function. Neuromuscul Disord 2002; 12: 484-493.

75 Khusnutdinova E, Gilyazova I, Ruiz-Pesini E, et al. A mitochondrial etiology of neurodegenerative diseases: evidence from Parkinson's disease. Ann NY Acad Sci 2008; 1147: 1-20.

76 Shoffner JM, Brown MD, Torroni A, et al. Mitochondrial DNA variants observed in Alzheimer disease and Parkinson disease patients. Genomics 1993; 17: 171-184.

77 Trifunovic A, Wredenberg A, Falkenberg M, et al. Premature ageing in mice expressing defective mitochondrial DNA polymerase. Nature 2004; 429: 417-423.

78 Harman D. Aging: a theory based on free radical and radiation chemistry. $J$ Gerontol 1956; 11: 298-300.

79 Migliaccio E, Giorgio M, Mele S, et al. The p66shc adaptor protein controls oxidative stress response and life span in mammals. Nature 1999; 402: 309-313.

80 Sun J, Folk D, Bradley TJ, et al. Induced overexpression of mitochondrial Mn-superoxide dismutase extends the life span of adult Drosophila melanogaster. Genetics 2002; 161: 661-672.

81 Schriner SE, Linford NJ, Martin GM, et al. Extension of murine life span by overexpression of catalase targeted to mitochondria. Science 2005; 308: 1909-1911.

82 Li Y, Huang TT, Carlson EJ, et al. Dilated cardiomyopathy and neonatal lethality in mutant mice lacking manganese superoxide dismutase. Nat Genet 1995; 11: 376-381.

83 Lebovitz RM, Zhang H, Vogel H, et al. Neurodegeneration, myocardial injury, and perinatal death in mitochondrial superoxide dismutase-deficient mice. Proc Natl Acad Sci USA 1996; 93: 9782-9787. 
84 Barazzoni R, Short KR, Nair KS. Effects of aging on mitochondrial DNA copy number and cytochrome c oxidase gene expression in rat skeletal muscle, liver, and heart. $J$ Biol Chem 2000; 275: 3343-3347.

85 Trounce I, Byrne E, Marzuki S. Decline in skeletal muscle mitochondrial respiratory chain function: possible factor in ageing. Lancet 1989; 1: 637-639.

86 Alexeyev MF, Ledoux SP, Wilson GL. Mitochondrial DNA and aging. Clin Sci (Lond) 2004; 107: 355-364.

87 Lenaz G. Role of mitochondria in oxidative stress and ageing. Biochim Biophys Acta 1998; 1366: 53-67.

88 Trifunovic A, Hansson A, Wredenberg A, et al. Somatic mtDNA mutations cause aging phenotypes without affecting reactive oxygen species production. Proc Natl Acad Sci USA 2005; 102: 17993-17998.

89 Di Lisa F, Bernardi P. Mitochondrial function and myocardial aging. A critical analysis of the role of permeability transition. Cardiovasc Res 2005; 66: 222-232.

90 Shigenaga MK, Hagen TM, Ames BN. Oxidative damage and mitochondrial decay in aging. Proc Natl Acad Sci USA 1994; 91: 10771-10778.

91 Grivennikova VG, Kareyeva AV, Vinogradov AD. What are the sources of hydrogen peroxide production by heart mitochondria? Biochim Biophys Acta 2010; 1797: 939-944.

92 Desler C, Lykke A, Rasmussen LJ. The effect of mitochondrial dysfunction on cytosolic nucleotide metabolism. J Nucleic Acids 2010; 701518.

93 Desler C, Munch-Petersen B, Stevnsner $\mathrm{T}$, et al. Mitochondria as determinant of nucleotide pools and chromosomal stability. Mutat Res 2007; 625: 112-124.

94 Brady NR, Hamacher-Brady A, Westerhoff HV, et al. A wave of reactive oxygen species (ROS)-induced ROS release in a sea of excitable mitochondria. Antioxid Redox Signal 2006; 8: 1651-1665.
95 Ussher JR, Lopaschuk GD. The malonyl CoA axis as a potential target for treating ischaemic heart disease. Cardiovasc Res 2008; 79: 259-268.

96 Levine B, Klionsky DJ. Development by self-digestion: molecular mechanisms and biological functions of autophagy. Dev Cell 2004; 6: 463-477.

97 Klionsky DJ, Emr SD. Autophagy as a regulated pathway of cellular degradation. Science 2000; 290: 1717-1721.

98 Simonsen A, Cumming RC, Brech A, et al. Promoting basal levels of autophagy in the nervous system enhances longevity and oxidant resistance in adult Drosophila. Autophagy 2008; 4 : 176-184.

99 Hara T, Nakamura K, Matsui M, et al. Suppression of basal autophagy in neural cells causes neurodegenerative disease in mice. Nature 2006; 441: 885-889.

100 Komatsu M, Waguri S, Chiba T, et al. Loss of autophagy in the central nervous system causes neurodegeneration in mice. Nature 2006; 441: 880-884.

101 Cuervo AM, Dice JF. How do intracellular proteolytic systems change with age? Front Biosci 1998; 3: D25-D43.

102 Cuervo AM, Bergamini E, Brunk UT, et al. Autophagy and aging: the importance of maintaining "clean" cells. Autophagy 2005; 1: 131-140.

103 Liesa M, Palacin M, Zorzano A. Mitochondrial dynamics in mammalian health and disease. Physiol Rev 2009; 89: 799-845.

104 Twig G, Elorza A, Molina AJA, et al. Fission and selective fusion govern mitochondrial segregation and elimination by autophagy. EMBO J 2008; 27: 433-446.

105 Bratic I, Trifunovic A. Mitochondrial energy metabolism and ageing. Biochim Biophys Acta 2010; 1797: 961-967.

106 Gottlieb RA, Carreira RS. Autophagy in health and disease. 5. Mitophagy as a way of life. Am J Physiol Cell Physiol 2010; 299: C203-C210. 